\title{
A Cluster Based Routing Algorithm MINIMIZING ENERGY CONSUMPTION IN WSN
}

\author{
Alaa Aadri and Najlae Idrissi \\ University of Sultan Moulay Slimane, Faculty of Sciences and Techniques \\ Information Processing Decision Support Laboratory \\ P.B. 523, Beni Mellal, Morocco
}

\begin{abstract}
Wireless sensor networks are continually being developed and constitute a new emerging technology that extends to many applications, they represent a very interesting field in the Internet of objects (IoT) and in information and communication technologies based on the manipulation of information through sensor nodes with limited characteristics (low storage capacity, autonomous source of energy, limited power), however, sensor nodes face the problem of energy constraints in terms of limited battery lifetime that has become the major issue in these networks and which represents a challenge to their design and management In these networks generally called WSN, Clustering routing represents a critical task to ensure energy efficiency and to overcome the constraints of congestion, collision and packet loss through several techniques of cluster formation and $\mathrm{CH}$ elections that have been proposed in literature. In this paper, we survey the principal clustering routing protocols in the literature, we describe their main characteristics and features, we propose a new cluster-based algorithm and we present its efficiency when comparing to some existing energy efficient clustering routing protocols using different simulation parameters.
\end{abstract}

\section{KEYWORDS}

WSN; IoT; Clustering; Energy efficiency; Data aggregation; performance analyzis

\section{INTRODUCTION}

In recent years WSN have attracted a lot of attention and have become an integral part of our lives, they are used in many civil and military applications where nodes can be deployed to capture, store, process, and transfer the sensed data permanently or also between physical contexts and virtual universes to help in operational decision-making. Routing issues and the selforganization of the nodes in the network are the two most studied themes in literature aiming to minimize energy consumed in the operations of capturing, processing and sending information via radio waves and to guarantee the overall functioning of the network. Clustering is one of the most efficient routing techniques to overcome the constraints imposed by the WSN environment and to achieve energy balancing in the network aiming to organize the network in clusters where each member node sends its collected data to its $\mathrm{CH}$ via the low-power radio link, which then aggregates them and sends them to the base station. This topology saves energy since only Cluster-Heads that are elected periodically so as not to exhaust the batteries of certain nodes quickly compared to others can transmit to the base station or to the sink node.

In the rest of this paper, section 2 gives brief state of the art of WSN, section 3 describes routing in WSN and a classification of the different routing protocols classes, section 4 presents our hierarchical routing protocol. And finally, section 5 shows simulation parameters and performance metrics we have used and presents results and discussions.

DOI: $10.5121 /$ ijwmn.2017.9405 


\section{State Of The Art Of Wireless Sensor Networks (WSN)}

\subsection{Presentation Of WSN}

A wireless sensor network consists of a set of electronic devices (sensors) capable of measuring physical values, processing them and transmitting them to a control center via a base station. Each sensor contains essentially four main units:

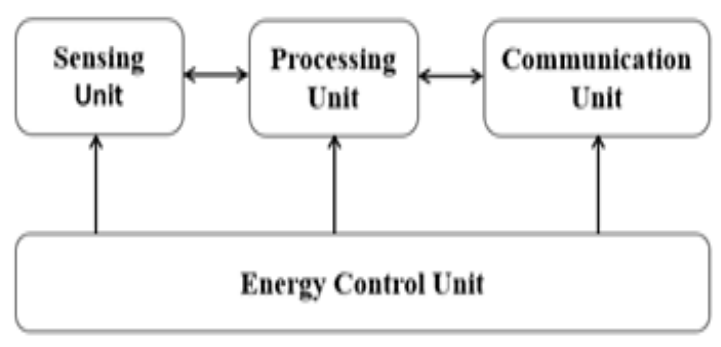

Figure 1. Sensor node components

- Sensing unit: usually consisting of a physical capture device that capture measurements and convert them into analog signals and of Analog-Digital Converters (ADCs) that convert these analog signals into digital signals.

- Processing unit: controls the procedures that enable the node to collaborate with other nodes to perform the acquisition tasks and store the collected data.

- Communication module: composed of a transceiver enabling communication between the different nodes of the network via a radio communication medium.

- Battery: the single source of energy that is generally neither rechargeable nor replaceable, it represents the main constraint while designing routing protocols for sensor networks.

\subsection{WSN APPLICATIONS}

Given their ability to monitor a variety of environmental phenomena including temperature, humidity, pressure, speed and direction of an object, ..., the WSN can be used in military application(Control, communication, calculation, intelligence, surveillance, recognition and targeting), in environment applications (control of environmental aspects that may affect crops, chemical and biological detection, pollution, fire, movements of species Animal health, etc.), in health application (integrated patient monitoring, and control of patients detect heartbeat and another for blood pressure), and in some commercial applications (interactive toys, monitoring of the equipment condition, control of products).

\subsection{Modelization OF WSN}

WSN can be modeled by a graph:

$$
G_{t=}\left(V_{t}, E_{t}\right) \quad(E q I)
$$


Where V represents the set of sensor nodes and $\mathrm{E}$ models all connections between these nodes. According to the organization of sensors in the deployment field, WSN can be presented under two main topologies:

- Flat topology: all the nodes are homogeneous and identical in terms of capacity and characteristics except the sink, which is responsible for the transfer of data collected to the end user. This topology allows high fault tolerance but it suffers from low scalability.

- Hierarchical topology: In this topology, nodes are divided into several levels of organization and responsibility. Clustering represents one of the most used methods; it aims to divide the network into clusters composed of a Cluster Head $(\mathrm{CH})$ and its cluster members that transfer their collected data for aggregation and transmission to the base station (BTS). This topology increases the scalability of the system, but it causes Cluster Heads overload and an unbalance in the energy consumption on the network.

\subsection{Constraints OF WSN}

Due to their specific characteristics, the factors that influence WSN factors that influence the design of WSN can be summarized as follows:

- Fault tolerance: the ability to maintain network functionality without interruptions in the presence of faults, this property $\mathrm{R}(\mathrm{t})$ is modelled in [1] by a Poisson distribution where $\mathrm{R}$ $(\mathrm{t})$ gives the probability of not having a failure for a sensor node during the time interval $[0, \mathrm{t}]$.

$$
\mathrm{R}(\mathrm{t})=\exp (-\lambda \mathrm{k} \mathrm{t})
$$

Where $\lambda \mathrm{k}$ is the failure rate of the sensor node $\mathrm{k}$, and $\mathrm{t}$ is the period of time.

- Scaling factor: monitoring of a phenomenon may require the deployment of a high number of nodes (hundreds or even thousands of sensors). The network must be able to exploit the highly dense nature of some applications.

A large number of nodes deployed in an application generates many transmissions and may impose difficulties for data transfer. The density can be calculated as follows:

$$
\mu(\mathrm{R})=(\mathrm{N} \Pi \mathrm{R} 2) / \mathrm{A} \quad(E q 3)
$$

Where $\mathrm{N}$ is the number of sensor nodes deployed in region $\mathrm{A}$, and $\mathrm{R}$ is the transmission range. $\mathrm{M}$ (r) then gives the number of nodes in the transmission range $\mathrm{R}$ of a given node in region $\mathrm{A}$.

- Deployment environment: due to the large number of nodes, it is difficult to configure them one by one. Therefore they must be able to self-organize themselves in order to route data to each other or to the Sink.

- Network topology: deployment, post-deployment (sensors can move or no longer operate) and redeployment of additional nodes to replace those that are defective or to meet the needs of tasks assigned to the network. This addition leads to the reorganization of the network and to the change of its topology

- Transmission media: since the nodes are connected by a wireless architecture and in order to enable operations on these networks, the transmission media must be standardized. Infrared, Bluetooth and Zig Bee radio communications are most commonly used.

- Energy consumption: the lifetime of a sensor depends essentially on the life of the battery. The energy consumed by a sensor is mainly due to the following operations: detection, processing and communication. 
International Journal of Wireless \& Mobile Networks (IJWMN) Vol.9, No.4, August 2017

- Limited physical security: WSN are based on wireless communications, therefore they are more vulnerable to attacks on the transmitted data. The conventional techniques used to deal with these attacks are not applicable in WSN due to their resource limitations (computing power and memory).

\subsection{ENERgY CONSUMPTION MODEL FOR COMMUNICATION IN WSN}

In the literature, an energy consumption model was proposed in [Heinzelman \& al, 2000] (Figure 2.) to describe the energy consumed by the sensors in each operation: the emission energy consumed to capture data and the communication energy that groups the transmission energy and the reception energy:

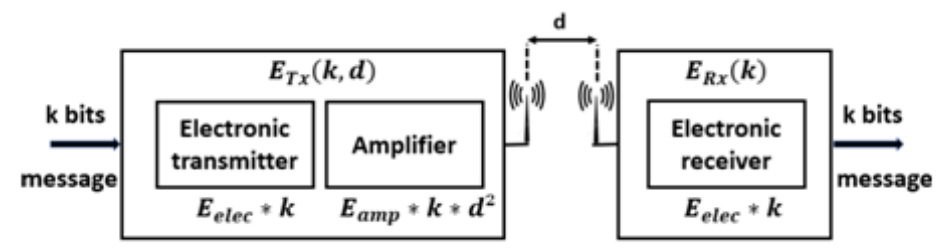

Figure 2. WSN Energy Consumption Module

- Transmitting energy: to transmit a message of $\mathrm{k}$ bits to a receiver far from $\mathrm{d}$ meters, the transmitter consumes:

$$
\begin{aligned}
& E_{T x}(k, d)=E_{T x \text { elec }}(k)+E_{T x \text { amp }}(k, d) \\
& E_{T x}(k, d)=\left(E_{\text {elec }} * k\right)+\left(E_{\text {amp }} * k * d^{2}\right)
\end{aligned}
$$

- Reception energy: to receive a message of $\mathrm{k}$ bits the receiver consumes:

$$
\begin{aligned}
& E_{R x}(k)=E_{R x \text { elec }}(k) \\
& E_{R x}(k)=E_{\text {elec }} * k
\end{aligned}
$$

Where $E_{\text {glec }}$ represents the electronic transmission energy and $E_{a m p}$ represents the amplification energy.

Both transmission and reception energies are determined by the amount of data to be communicated, by the transmission distance and by the physical properties of the radio module.

\subsection{Radio Module Status}

The radio module is the component that ensures communication between the nodes of the network and therefore it consumes the big part of energy, It can have four states: active, reception, transmission and sleep:

- Active state: the radio is on but the sensor node is neither receiving nor transmitting. This state causes a loss of energy due to unnecessary listening of the transmission channel.

- Sleep status: the radio is turned off.

- Transmission status: the radio transmits a packet.

- Receiving status: the radio receives a packet. 


\section{ROUTING IN WSN}

Data routing designs the way how information is routed to its destination through a network connection, it consists on optimal packets delivering through the network using the least possible resources and ensuring network fault tolerance. In this part, we present a classification of WSN routing protocols with a focus on those based on the network hierarchization and on which a performance study will be applied in the next part of this article.

\subsection{Classification Of WSN Routing Protocols}

Data Routing have attracted a lot of interest among the researchers, many routing protocols have been presented depending on type of application and on data routing strategies.

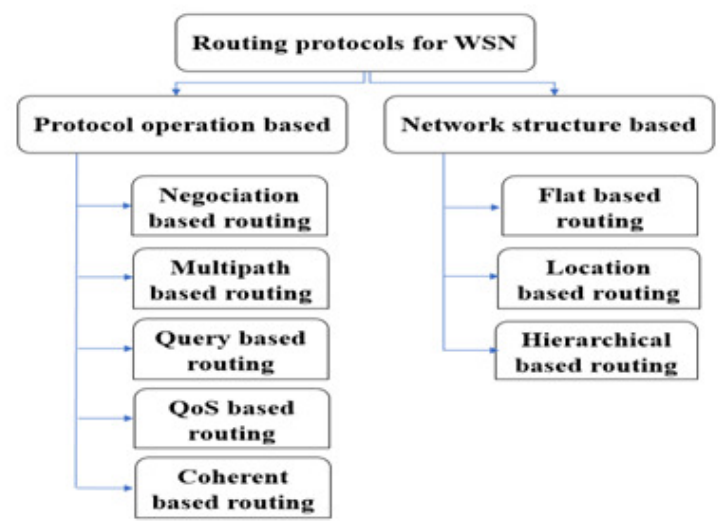

Figure 3. WSN Routing protocols classification

As shown in Figure 3. WSN routing protocols in WSN can be divided to:

- Negotiation-based routing: routes selection is based on the available resources to eliminate the redundancy of data in the network.

- Multipath-based routing: multi paths are used rather than single paths, which increases the fault tolerance but also energy consumption and traffic generation.

- Query-based routing: the destination node propagates a request for particular information in the network and the nodes possessing it respond by sending it to the requesting node.

- QOS-based routing: QoS-aware protocols consider end-to-end delay requirements while setting up the routes in the sensor network.

- Coherent-based routing: forwards data after processing and redundancies elimination to the aggregator nodes in order to improve energy efficiency

- Flat-based routing: In this routing scheme nodes are identical (in terms of battery and hardware complexity), the disadvantage is that scalability becomes critical for a very large number of sensor nodes, hence the need to manage and organize the nodes using access control medium (MAC).

Location-based routing: uses location information to guide route discovery and data transmission. It allows an optimized routing at reduced cost but the disadvantage is that each node must know the location of the other nodes of the network. 


\subsection{HiERARCHICAL RoUTING IN WSN}

Hierarchical-based routing represents one of the most efficient strategy to improve energy efficiency and to achieve self-organization of the network. Several routing strategies have been proposed in the literature, in this paper we focus on the following energy efficient hierarchical routing protocols:

\subsubsection{LEACH (Low-Energy Adaptive Clustering Hierarchy)}

This protocol was proposed by ChandraKasan \& al. to provide an efficient solution to the problem of energy consumption in the WSN.

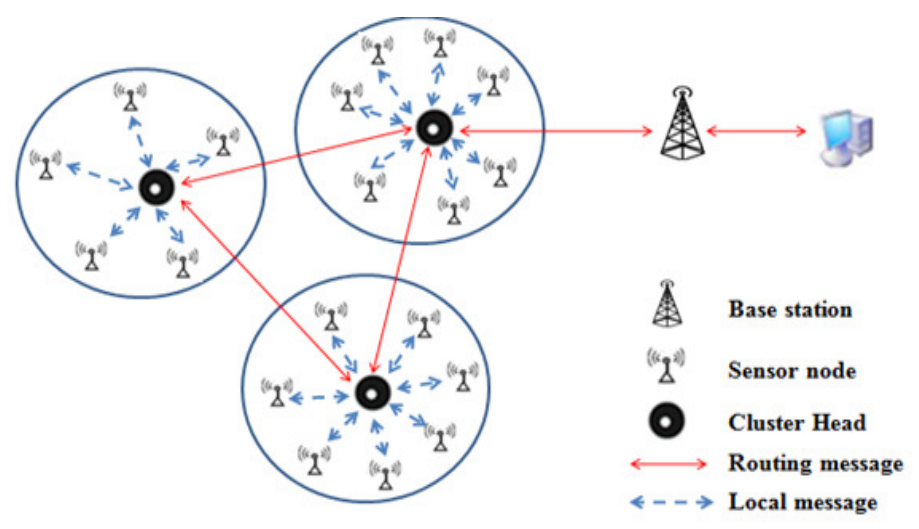

Figure 4. Scheme of LEACH protocol

LEACH is based on the formation of clusters in which the elected $\mathrm{CHs}$ collect and aggregate the data captured by their cluster member nodes to subsequently transmit them to the base station, a $\mathrm{CH}$ performs its role as cluster leader for a period of time called "round", at the beginning of each round each node of the network determines whether it wants to be a $\mathrm{CH}$ by calculating a number between 0 and 1 if this number is less than a threshold $\mathrm{T}(\mathrm{n})$ the node becomes $\mathrm{CH}$, the threshold $\mathrm{T}(\mathrm{n})$ is expressed by the relation:

$$
T(n)=\left\{\begin{aligned}
\frac{p}{1-p\left[\operatorname{rmod}\left(\frac{1}{p}\right)\right]} & \text { if } n \in G \\
0 & \text { if not }
\end{aligned}\right.
$$

With p: the percentage of CHs in the network; r: the number of the current round; G: the number of nodes that have not been selected as $\mathrm{CHs}$ in the previous $1 / \mathrm{p}$ rounds. Once the clusters are formed, each $\mathrm{CH}$ sends its identifications to the nodes of the network through the CSMA protocol and assigns to each member node of its cluster an interval of time during which it can send its data based on the TDMA approach.

\subsubsection{PEGASIS (Power-Efficient Gathering In Sensor Information Systems)}

This routing protocol is considered as an optimization of LEACH, it gathers the network nodes in a long chain based on the principle that a node can communicate only with the closest node to it. Thus, each node adjusts its radio for a very short communication to conserve its energy. To communicate with the Sink, the process is organized into rounds; during each round a single node is allowed to communicate directly with the sink. This privilege is granted to all the nodes of the network in turn. A better conservation of energy is obtained by the data aggregating on each node 
of the network: in each round, only one node can communicate directly with the sink, it's called "leader node", this privilege is given in turn to all the nodes of the network. Nodes transmit their data throw their neighboring nodes toward the leader node that sends it afterward to the base station.

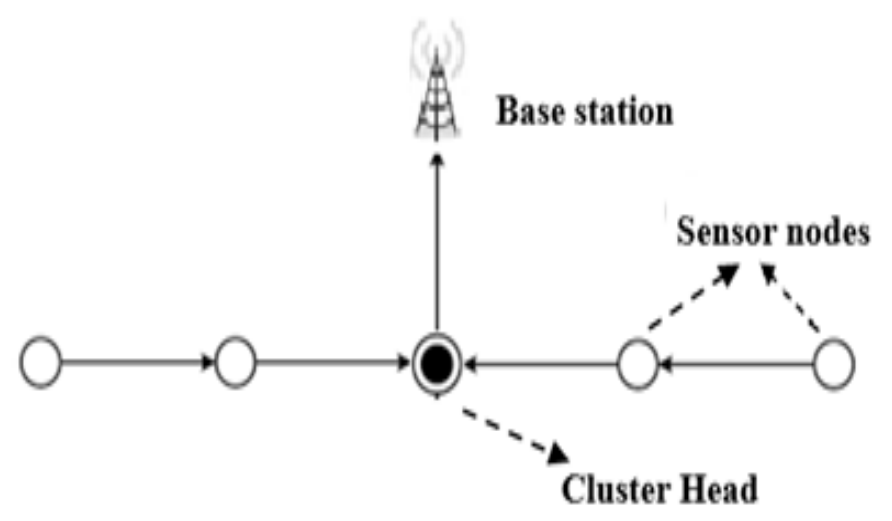

Figure 5. Chain construction in PEGASIS

\subsubsection{HEED (Hybrid Energy-Efficient Distributed Clustering)}

This protocol aims to divide the network into one hop clusters jump where $\mathrm{CHs}$ are elected according to two metrics: energy and cost of paths, each node calculates its probability to become $\mathrm{CH}$ by the following formula:

$$
C H_{\text {prob }}=C_{\text {prob }} *\left(E_{\text {Res }} / E_{\text {Max }}\right)
$$

Where $E_{\text {Res }}$ represents the remaining energy of the node and $E_{M a x}$ its initial energy. $C H_{\text {prob }}$ is always greater than a threshold $p_{\min }$ Inversely proportional to $E_{\operatorname{Max}}$.

A node of the network can be presented under two states: "tentative status" if $\mathrm{CH}_{\text {prob }}<1$ or "final status" if $\mathrm{CH}_{\text {prab }}=1$.

\section{Proposed Cluster-Based Routing Algorithm For WSN}

\subsection{DESCRIPTION}

Our hierarchical routing algorithm intends to form interconnected clusters through discovery messages exchange between the nodes and to use a TDMA technique to set a sleeping mechanism for sensors in order to extend the network lifetime, it integrates also a method for data aggregation based on multi-hops intra-cluster and inter-cluster communications aiming to satisfy the compromise "Energy consumption - Quality of Service (QoS)".

\subsection{Phases Of Our Routing Algorithm}

The main objective of the proposed protocol is energy consumption minimization and therefore prolongs the lifetime of the network; flow chart shown in the Figure 5. shows the description of the first round process in our cluster-based algorithm: 
International Journal of Wireless \& Mobile Networks (IJWMN) Vol.9, No.4, August 2017

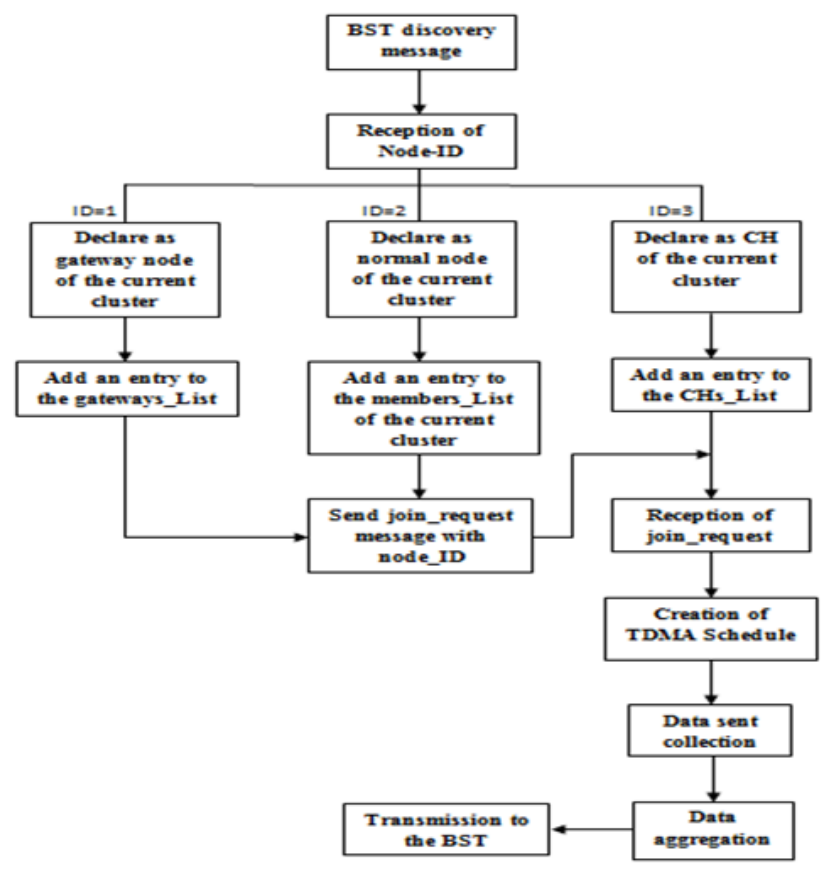

Figure 6. Flow chart of the set up phase in our routing algorithm

\subsubsection{Initialization Phase}

Initially the nodes are in listening mode, the clusters will be defined by a diffusion technique controlled by the BTS which broadcasts a discovery message on the covered zone containing a node identifier fixed at ' 0 ' and incremented each time by the receiver node before sending it to its neighbours, and also a cluster identifier incremented only by the $\mathrm{CH}$ nodes, these identifiers are manipulated so as to construct clusters with 2 hops gradually with the $\mathrm{CH}$ node as a center.

\subsubsection{Clustering Phase}

It is well known that in a sensor network (multi-hop) $\mathrm{CH}$ election affects on the energy consumption in each clustering method, our proposed approach tends to reduce this effect by using an efficient clustering method. We denote by $n$ a single node in the network and by $N$ the total number of the nodes in the network, the pseudo code of clusters construction phase:

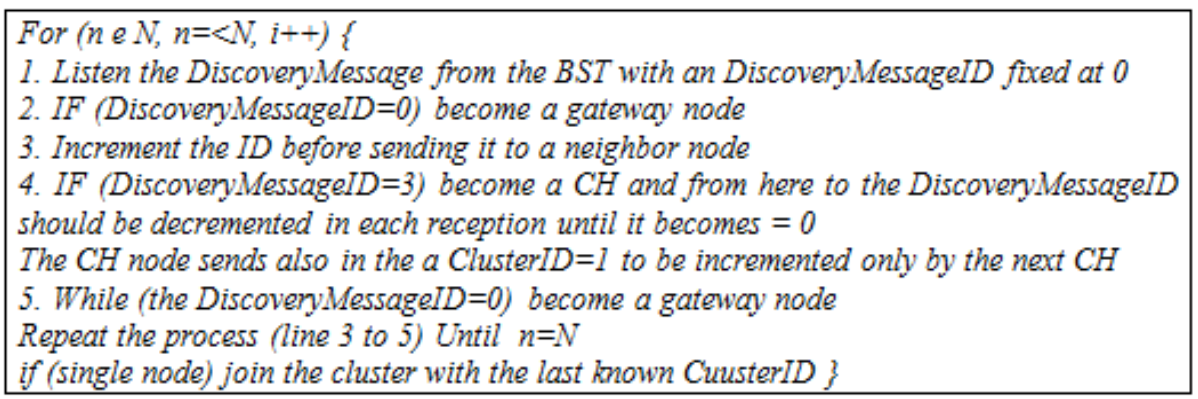

In the end of this phase, three lists are formed:

- Members_List: defined in each $\mathrm{CH}$, contains the set of member nodes of a cluster.

- Gateways_List: defined in each $\mathrm{CH}$, gives an overall view of neighboring clusters.

- CHs_List: defined in the BST, list of CHs in the network. 
Once the clusters are formed, $\mathrm{CHs}$ receive data from simple nodes and creates TDMA tables based on the number of nodes that form the clusters. When all the data are received, they are compressed by the $\mathrm{CHs}$ and transmitted to the base station.

\subsubsection{TDMA SCHEMA AND ROUTE MANAGEMENT}

Once the clusters are formed, the Base station uses a Msg_BTS to assign a MAC transmission code to each cluster in order to limit inter-cluster collisions, in their turn $\mathrm{CHs}$ assign a MAC transmission code to each member node to limit intra-cluster collisions. In addition, in each cluster a list of all the gateway nodes to the neighbouring clusters is built and will be used in inter-cluster routing.

A route is defined by the triplet (SourceID, DestinationID, cost). Intra-cluster communications are operated simultaneously in all clusters of the network. For inter-cluster communication process, each $\mathrm{CH}$ broadcasts a message which contains its ID, residual energy, the number of his cluster members, and distance to the base station.

\subsubsection{Re-ELECTION OF CH AND THE METRIC USED}

In each new round the election of the new $\mathrm{CH}$ doesn't pass through the BST but rather through the old $\mathrm{CH}$ according to three elements:

- Energy level of the new $\mathrm{CH}$.

- Density of the nodes at two hops.

- Distance from the old $\mathrm{CH}$.

Each node calculates its probability to be a $\mathrm{CH}$ using the fallowing metric:

$$
\left.\mathrm{P}(\mathrm{CH})=\left[\alpha^{*} \text { (Res-energy) }+\beta *(2 \text {-Density })+\gamma * \text { (Distance from last } \mathrm{CH}\right)\right] \quad(E q 7)
$$

Where the 2-Density refers to the number of neighbouring nodes at two two-hop and the distance between two nodes $(i)$ and $(j)$ is denoted by:

$$
\operatorname{Distance}_{(i, j)}(t)=\sqrt{\left(X_{i}(t)-X_{i}(t)\right)^{2}+\left(Y_{i}(t)-Y_{j}(t)\right)^{2}}
$$

The values of the parameters $(\alpha, \beta, \gamma)$ depend on the application with $: \alpha+\beta+\gamma=1$

This approach avoids voting consumption, reduces the energy of clustering and assures $\mathrm{CH}$ rotation which helps to prolong network lifetime.

\subsubsection{Data Aggregation MODEL}

Our aggregation method is based on the Data centric approach used to solve the data implosion problem that characterizes the sensor networks enabling processing of redundant data at the time of transmission on the network.

In each cluster, sensor nodes of a level $(\mathrm{N})$ aggregate the data coming from the lower level nodes $(\mathrm{N}-1)$. In addition, a data aggregation process is performed between $\mathrm{CHs}$ during the inter-clusters communications insured by the gateway nodes. 


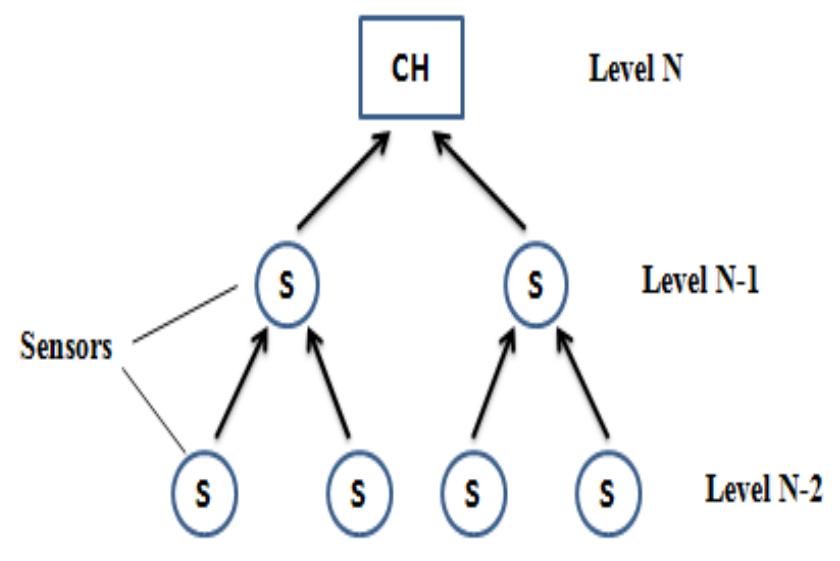

Figure 7. Data aggregation process in each cluster

The data aggregation on several levels represents an effective way to reduce the amount of information communicated and therby to decrease energy consumption and to improve network lifetime.

\subsubsection{EXChANGED PACKETS}

Our protocol uses three types of packets: HelloPacket to discover neighbouring nodes and establish paths, ClusterConstructPacket used in the construction phase of the clusters and in the election of CHs, DataPacket that contains the data to be transmitted in the network. Depending on the packet type, different informations are used in our protocol:

- Identifier of the source node, its $\mathrm{CH}$ and its coordinates (x, y)

- Identifier of the parent of the source node

- Calculated metric of the source node

- Number of hops between the source node and its $\mathrm{CH}$

- List of the source node neighbors and their coordinates

- List of the neighboring clusters addresses

- Identifier of the destination node and its ClusterID (DestinationClusterID)

- NexthopID : Nexthop node identifier

- Data: Transmitted data

- TTL Lifetime of the packet

\section{RESULTS AND DISCUSSIONS}

In this part, we compare our routing protocol with some efficient hierarchical routing protocols from the literature: LEACH, PEGASIS and HEED, and we present the results of our simulation based on evaluating metrics of energy level, throughput and packet delivery ratio.

\subsection{Simulation PARAMETERS}

In our simulation, we distribute the sensors in the capture field with a sufficient deployment density to ensure connectivity on the network, the nodes are homogeneous and possess the same transmission radius and can send their data periodically. Our simulations have been run in MATLAB (Matrix laboratory), Table 1 lists the simulation parameters. 
International Journal of Wireless \& Mobile Networks (IJWMN) Vol.9, No.4, August 2017

Table I. Simulation Parameters

\begin{tabular}{|c|c|}
\hline Parameters & Values \\
\hline Simulation area & $100 \mathrm{~m} \mathrm{X} 100 \mathrm{~m}$ \\
\hline Initial energy E0 & $0,5 \mathrm{~J}$ oules \\
\hline $\begin{array}{c}\text { Energy consumed in the } \\
\text { electronics circuit, Eelec }\end{array}$ & $50 \mathrm{~nJ} / \mathrm{bit}$ \\
\hline $\begin{array}{c}\text { Energy consumed by the } \\
\text { amplifier Efs }\end{array}$ & $10 \mathrm{pJ} / \mathrm{bit} / \mathrm{m}^{2}$ \\
\hline Base station location & $\mathrm{X}=0 \mathrm{~m} ; \mathrm{Y}=0 \mathrm{~m})$ \\
\hline Number of nodes & 100 \\
\hline Sensing rage & $5 \mathrm{~m}$ \\
\hline Transmission range & $50 \mathrm{~m}$ \\
\hline Message size & $2000 \mathrm{bits}$ \\
\hline Bandwidth & $5000 \mathrm{bits} / \mathrm{sec}$ \\
\hline Control packet & $250 \mathrm{bits}$ \\
\hline Data transmission speed & $100 \mathrm{bits} / \mathrm{sec}$ \\
\hline
\end{tabular}

\subsection{ReSUlts AnAlysis}

In this part, we present our simulation results and we compare the performance of our clusterbased routing protocol with the studied clustering protocols.

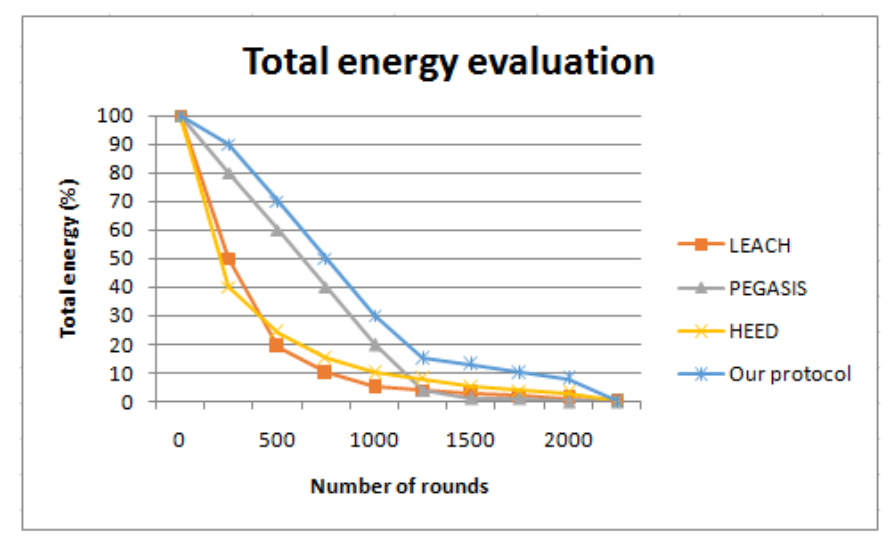

Figure 8. Total energy evaluation in our protocol Vs LEACH, PEGASIS and HEED

The total energy in each round is presented by the sum of residual energy of all the network nodes, the rotation of the $\mathrm{CH}$ role on all the nodes of the cluster allows to balance the energy consumption of the cluster, but in the meanwhile it generates an overconsumption of energy since each rotation of $\mathrm{CH}$ requires a diffusion phase to inform the nodes about the new $\mathrm{CH}$.

PEGASIS uses only one transmission to the BS per round and reduces the control overhead which improves its performance. In LEACH, the ability to communicate with the sink through any node on the network requires considerable energy consumption from distant nodes, so the aggregation of the data is centred at the level of the CHs, which makes them the weak links of the network. PEGASIS considers that all the nodes of the network can reach the sink, which requires an adjustable transmission with an important energy overhead.

Figure 8. shows that the energy decrease in our protocol is much slower than the other protocols (LEACH, PEGASIS and HEED) because it selects $\mathrm{CH}$ nodes according to the residual energy and to the minimal energy from the previous $\mathrm{CH}$ rather than random probability and that extends network lifetime. 


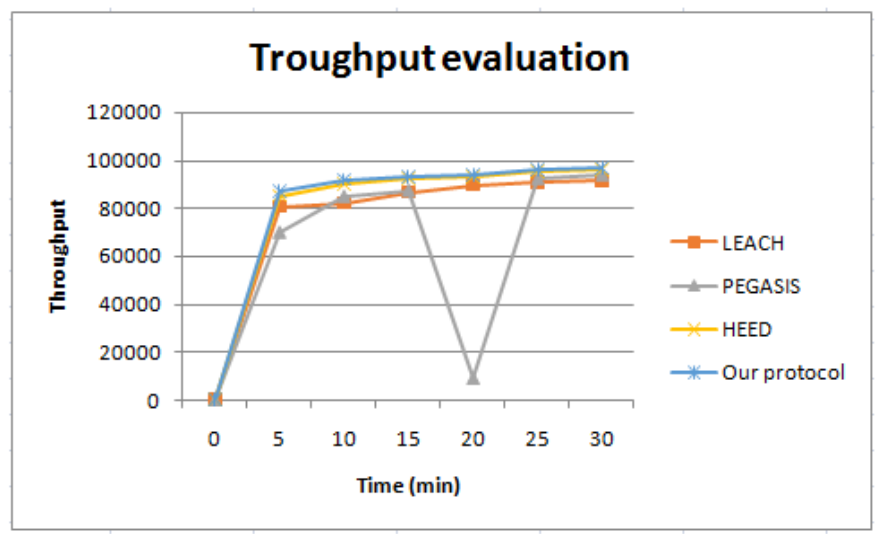

Figure 9. Throughput evaluation in our protocol Vs LEACH, PEGASIS and HEED

The throughput is the ratio of the number of packets sent to the total number of packets, the greater value of throughput means the better performance of the protocol.

As it can be seen from Figure 9. our protocol has an efficient throughput when compared with the other protocols. Since our protocol is executed in cooperation between all the nodes of the network, each node decides independently of its role which guarantees better distributed energy consumption. Moreover, the use of multi-hops routing guarantees a compromise between the size of the clusters and the number of clusters in the network which ensures energy balancing.

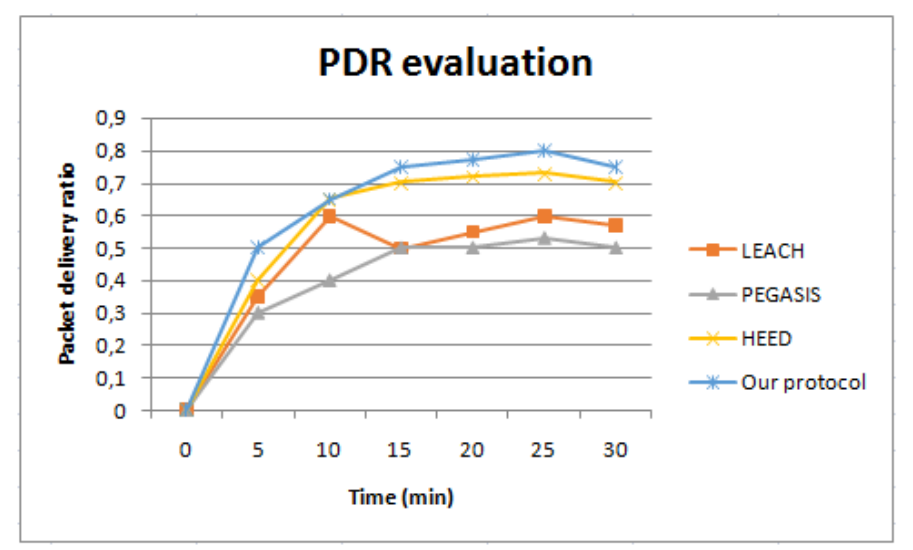

Figure 10. Packet delivery ratio evaluation in our protocol Vs LEACH, PEGASIS and HEED

The packet delivery ratio is the number of packets delivered to the destination to the number of packets supposed to be received, the greater value of packet delivery ratio means the better performance of the protocol.

In LEACH, energy consumption is shared across all nodes and the use of TDMA / CDMA techniques allows for a hierarchy and multi-level clustering, which saves more energy.

PEGASIS protocol try to eliminate of the overhead caused by dynamic cluster formation in LEACH but the length of the chain formed in PEGASIS protocol can considerably increase the data delivery time and the only node allowed to communicate with the BS can become a bottleneck within the network. 
From Figure 10. we can notice that our protocol provides better Packet Delivery Ratio due to its ability to find rapidly the next hop with minimum transmission distance comparing to the others routing schemes

The proposed cluster-based routing algorithm in this paper brings a contribution to the resolution of encountered problems in WSN, particularly that of energy consumption which has been the challenge in this field, based on the identification of the sources of difficulties that other hierarchical routing protocols suffer from through a performance analysis, it benefits from two key designs: our node discovery process and the limitation of transmission radius that avoids collisions and requires less transmission power.

\section{Conclusions}

WSN represent the fusion of two modern computing poles: embedded systems and wireless communications, they must guarantee good Quality of Service and should integrate mechanisms and techniques that enable energy conservation to prolong the lifetime of the entire network and particularly during the data routing phase. In this paper, we proposed a new cluster-based energy efficient routing algorithm which we compared to some of the existing hierarchical energy efficient routing protocols in terms of throughput, packet delivery ratio and energy consumption that represents the ultimate challenge for researchers in the WSNs field. The obtained results has shown that our routing algorithm is more optimal and energy efficient. As a future work, we intend to develop our protocol to guarantee its efficiency with other parameters by taking into consideration more WSN specifications; we will also improve its quick adaptation to topological and parametric changes and address issues such as Quality of Service (QoS) to achieve the highest efficiency in energy consumption.

\section{REFERENCES}

[1] W. R. Heinzelman, et al., (2010) "Energy-efficient communication protocol for wireless microsensor networks," 33rd Annual Hawaii International Conference on System Sciences.

[2] S. Lindsey, C. Raghavendra, (2002) "PEGASIS: Power-Efficient Gathering in Sensor Information Systems", IEEE Aerospace Conference Proceedings, Vol. 3, 9-16 pp. 1125-1130.

[3] Gang Lu et al, (2008) "An Adaptive Energy-Efficient and Low-Latency MAC for Data Gathering in Sensor Networks", 2004. 9. Injong Rhee et al, "Z-MAC: a Hybrid MAC for Wireless Sensor Networks", IEEE/ACM Transactions on Networking (TON), Vol 16, Issue 3.

[4] R. C. Shah and J. Rabaey, "Energy Aware Routing for Low Energy Ad Hoc Sensor Networks", IEEE Wireless Communications and Networking Conference (WCNC), March 17-21, 2002, Orlando, FL.

[5] Ossama Younis and Sonia Fahmy "Distributed Clustering inAd-hoc Sensor Networks: A Hybrid, Energy-Efficient Approach for Ad-hoc Sensor Networks", Department of Computer Sciences, Purdue University.

[6] Falko Dressler and Ozgur B. Akan, (2010) "A survey on bio-inspired networking", The International Journal of Computer and Telecommunications Networking, vol. 54, issue 6, pp. 881-900.

[7] S. Banerjee and S. Khuller, A clustering scheme for hierarchical control in multi-hop wireless networks, in Proceedings of 20th Joint Conference of the IEEE Computer and Communications Societies.

[8] Dahlila P. Dahnil, Yaswant P. Singh and Chin Kuan Ho, (2011)“Energy-Efficient Cluster Formation in Heterogeneous Wireless Sensor Networks: A Comparative Study”, Feb. 13 16, ICACT.

[9] Vishal Kumar Arora, Vishal Sharma, Monika Sachdeva (2016) “ A survey on LEACH and other's routing protocols in wireless sensor network", Optik - International Journal for Light and Electron Optics, Volume 127, Issue 16.

[10] Abbas Nayebi, Hamid Sarbazi-Azad, (2011) “Performance modeling of the LEACH protocol for mobile wireless sensor networks", Journal of Parallel and Distributed Computing, Volume 71, Issue 6, Pages 812-821. 
[11] Sudhanshu Tyagi, Neeraj Kumar, (2013) “A systematic review on clustering and routing techniques based upon LEACH protocol for wireless sensor networks", Journal of Network and Computer Applications, Volume 36, Issue 2, Pages 623-645

[12] Vrinda Gupta, Rajoo Pandey, (2016) “ An improved energy aware distributed unequal clustering protocol for heterogeneous wireless sensor networks", Engineering Science and Technology, an International Journal, Volume 19, Issue 2, Pages 1050-1058.

[13] Shijun He, Yanyan Dai, Ruyan Zhou, Shiting Zhao, (2012) “ A Clustering Routing Protocol for Energy Balance of WSN based on Genetic Clustering Algorithm”, IERI Procedia, Volume 2, Pages 788-793.

[14] M. Mehdi Afsar, Mohammad-H. Tayarani-N “ Clustering in sensor networks: A literature survey”, Journal of Network and Computer Applications, Volume 46, November 2014, Pages 198-226.

[15] Md Azharuddin, Pratyay Kuila, Prasanta K. Jana, (2015) "Energy efficient fault tolerant clustering and routing algorithms for wireless sensor networks ", Computers \& Electrical Engineering, Volume 41, Pages 177-190.

[16] Ramnik Singh, Anil Kumar Verma, (2017) "Energy efficient cross layer based adaptive threshold routing protocol for WSN", International Journal of Electronics and Communications, Volume 72, Pages 166-173.

[17] Shilpa Mahajan, Jyoteesh Malhotra, Sandeep Sharma, (2014) “ An energy balanced QoS based cluster head selection strategy for WSN", Egyptian Informatics Journal, Volume 15, Issue 3, Pages 189-199.

[18] Wang Ke, Ou Yangrui, Ji Hong, Zhang Heli, Li Xi, (2016) "Energy aware hierarchical cluster-based routing protocol for WSNs", The Journal of China Universities of Posts and Telecommunications, Volume 23, Issue 4.

[19] Melike Yigit, V. Cagri Gungor, Etimad Fadel, Laila Nassef, Nadine Akkari, Ian F. Akyildiz, (2016) "Channel-aware routing and priority-aware multi-channel scheduling for WSN-based smart grid applications" Journal of Network and Computer Applications, Volume 71, Pages 50-58.

[20] Hang Shen, Guangwei Bai, (2016) "Routing in wireless multimedia sensor networks: A survey and challenges ahead", Journal of Network and Computer Applications, Volume 71, Pages 30-49.

[21] Jalel Ben-Othman, Bashir Yahya, (2010) "Energy efficient and QoS based routing protocol for wireless sensor networks", Journal of Parallel and Distributed Computing, Volume 70, Issue 8, Pages 849-857.

[22] S. Abarna, N. Arumugam, (2014)" Performance Comparison of Various Energy Aware Protocols for WSN Applications", International Journal of Innovative Research in Electronics and Communications (IJIREC) Volume 1, Issue 9, PP 1-8.

[23] Nathalie Mitton and Eric Fleury, (2005) "Efficient broadcasting in self-organizing multi-hop wireless networks In ADHOC-NOW, pages 192-206.

\section{Authors}

Alaa Aadri PhD student and member of the TIAD Laboratory in the Faculty of Sciences and Techniques, Sultan Moulay Slimane University. Obtained a master degree in business intelligence in 2013, completed by a master degree in Information Systems Management, Institute of Business Administration IAE-Lyon - France in 2015. His current interests include Mobile Computing, Networking and intelligent Systems developing.

Najlae Idrissi Member the TIAD Laboratory and professor in the Faculty of Sciences and Techniques, Sultan Moulay Slimane University in Beni mellal - Morocco. His current interests include Mobile computing and networking,signal,image and video processing and datamining.
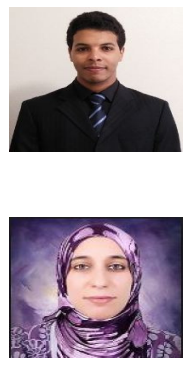